\title{
Synthesis of novel conjugated enynes: a reaction of lithium acetylenides with $\beta$-dimethylaminovinyl ketones
}

\author{
Marcos A. P. Martins, * Marcelo Rossatto, Fernanda A. Rosa, Pablo Machado, \\ Nilo Zanatta, and Helio G. Bonacorso
}

Núcleo de Química de Heterociclos (NUQUIMHE), Departamento de Química, Universidade Federal de Santa Maria, 97105-900 Santa Maria, RS, Brazil

E-mail:.mmartins@base.ufsm.br

\begin{abstract}
The reaction of lithium acetylenide $(\mathrm{R}-\mathrm{C} \equiv \mathrm{CLi}$, where $\mathrm{R}=\mathrm{Ph}$, Pentyl) with $\beta$-dimethylamino vinyl ketones $\left[\mathrm{X}-4-\mathrm{Ph}-\mathrm{C}(\mathrm{O}) \mathrm{CH}=\mathrm{CH}-\mathrm{NMe}_{2}\right.$, where $\left.\mathrm{X}=\mathrm{F}, \mathrm{Cl}, \mathrm{Br}, \mathrm{NO}_{2}\right]$ in the presence of $\mathrm{BF}_{3} \bullet \mathrm{OEt}_{2}$ is described. The regiospecific formation of $(E)$-1-aryl-2-alken-4-yn-1-ones, the 1,4addition products, was observed in moderate to good yields.
\end{abstract}

Keywords: Enynes, acetylenes, enaminones, organolithium

\section{Introduction}

Conjugated enynes are fundamental building blocks in organic synthesis. ${ }^{1}$ These moieties are found to be integral parts of highly potent antitumor, ${ }^{2}$ and antifungal agents, ${ }^{3}$ and first line components of antiretroviral therapy. ${ }^{4}$ Terbinafine $\left(\text { Lamisil }{ }^{\mathrm{TM}}\right)^{3}$ is one example of an enyne derivative in clinical use. In addition, in many systems rigidification has been an efficient method used to increase the activity of a drug or to reduce its side-effects. This strategy locks the drug molecule into a more rigid conformation such that it cannot take up other shapes or conformations. One such method is to incorporate a rigid functional group such as an alkyne in the drug candidates. ${ }^{5}$

The synthesis of enynes has received special attention during recent decades, and new methods for their preparation have been developed. The most employed protocol for the synthesis of such compounds has been the palladium-catalysed cross-coupling reactions between vinyl halides and terminal acetylenes. ${ }^{6}$

On the other hand, the reactions of acetylenes with $\alpha, \beta$-unsaturated ketones have been scarcely reported. With regards to $\beta$-dimethylaminovinyl ketones, Mellor et al. ${ }^{7}$ demonstrated the reaction of 4-dimethylamino-1,1,1-trifluoro-3-buten-2-one with alkyl(aryl)acetylenes based on Grignard and organolithium reagents. In these reactions only a single example in moderate 
yield (37\%) was reported using organolithium reagents. Martins et al. ${ }^{8}$ also reported the preparation of a series of halo-enynes with good yields, which have been used for the preparation of heterocycles.

In recent years, we have developed a general synthesis for a large number of 1,1,1-trihalo-4methoxy-3-alken-2-ones ${ }^{9,10}$ important halogen-containing building blocks and have demonstrated their usefulness in heterocyclic preparations, for example, isoxazoles, pyrazoles, pirazolium chlorides, pyrrolidinones, pyrimidines, pyridines, thiazines and diazepines. ${ }^{10,11}$ In the continuation of our program to develop new halogen-containing building blocks, we are developing a methodology to synthesize a series of halo-enynes. Since the conjugated enynes are found to be important building blocks and essential units in a variety of biologically active compounds, the aim of this work is to report the synthesis of a series of novel conjugated enynes from the reaction of lithium acetylenide with $\beta$-dimethylaminovinyl ketones under Lewis acid promotion (Scheme 1).

\section{Results and Discussion}

Enaminones are versatile synthons for the synthesis of a variety of compounds, and their preparation has been recognized for some time. The convenient synthesis of $\beta$ dimethylaminovinyl ketones from the reaction of aryl ketones and formamide acetal has already been reported. ${ }^{12}$ In this study, the $\beta$-dimethylaminovinyl ketones 3 were prepared in good yields $(68-98 \%)$ by the one-step reaction between substituted ketones, N,N-dimethylformamide dimethyl acetal and boron trifluoride diethyl etherate $\left(\mathrm{BF}_{3} \bullet \mathrm{OEt}_{2}\right)$ in toluene under reflux for 24 hours. $^{12,13}$ The enaminones were obtained in $(E)$-configuration because the coupling constant was around ${ }^{3} J_{\mathrm{H} 3-\mathrm{H} 4}=12 \mathrm{~Hz}$. The structures of 3a-d were confirmed by NMR spectroscopy $\left({ }^{1} \mathrm{H}\right.$ and ${ }^{13} \mathrm{C}$ ) and MS spectra.

The synthesis of compounds 4a-d and 5a-d was carried out from the reaction of acetylene $\mathbf{1}$ with butyl lithium and the subsequent reaction with the corresponding electrophile agent $\mathbf{3}$, in the presence of 2 mol-equiv of $\mathrm{BF}_{3} \bullet \mathrm{OEt}_{2}$ (Scheme 1). Firstly, the lithium acetylenides were generated from the respective acetylenes 1,2 using BuLi in THF, at $-20{ }^{\circ} \mathrm{C}$ for $30 \mathrm{~min}$. In a second step, the reaction between $\beta$-dimethylaminovinyl ketones $\mathbf{3}$ and lithium acetylenides was carried out using $\mathrm{BF}_{3} \bullet \mathrm{OEt}_{2},-15{ }^{\circ} \mathrm{C}$ for 2 hours, and THF as solvent. The transformation was completed after being stirred at room temperature for 16 hours. Under this condition, a regiospecific 1,4-addition was observed, furnishing the products (4a-d, 5a-d) in 63-80\% yield. This finding is in accordance with a previous report from the literature, in which organolithium reagents were preferentially added to $\alpha, \beta$-unsaturated ketones in a 1,4-mode. Moreover, few cases can be found of 1,2-addition to the carbonyl-carbon using these reagents. ${ }^{14}$ The charge controlled 1,2-addition and orbital controlled 1,4-addition theories have been used to explain the addition reaction of organometallic reagents with $\alpha, \beta$-unsaturated carbonyl compounds. ${ }^{7,15}$ Organolithium reagents (hard nucleophiles) react by a 1,2-addition, and organocopper reagents 
(soft nucleophiles) react by a 1,4-addition. ${ }^{7,15}$ The reactivity of Grignard reagents is situated between that of the organolithium and organocopper reagents. In the reaction conditions used in our laboratory, the results show that the reaction of $\mathbf{1 , 2}$ with 3 favored the formation of 1,4addition products. We believe that, for these substrates, the synthesis of 1,4-addition products, was additionally favored because the organolithium reagents were used in the presence of boron trifluoride diethyl etherate. This fact led to the formation of an alkynyl trifluorborate, a soft nucleophile, ${ }^{16}$ as shown in Scheme 1.
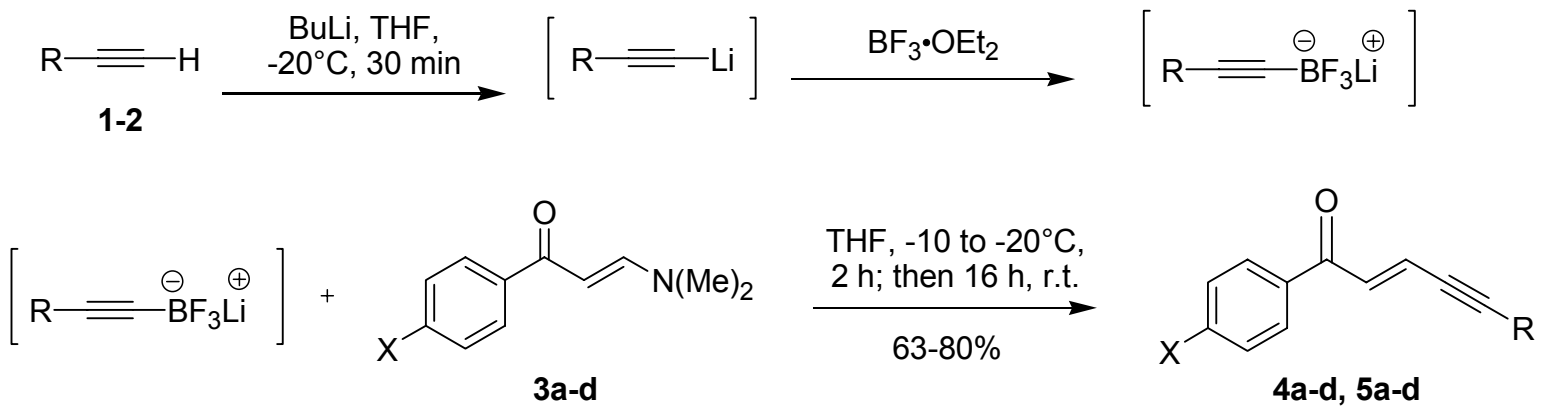

\begin{tabular}{cccc}
\hline Product & $\mathrm{R}$ & $\mathrm{X}$ & Yield (\%) \\
\hline 4a & Pentyl & $\mathrm{F}$ & 70 \\
4b & Pentyl & $\mathrm{Cl}$ & 75 \\
4c & Pentyl & $\mathrm{Br}$ & 78 \\
4d & Pentyl & $\mathrm{NO}_{2}$ & 65 \\
\hline
\end{tabular}

\begin{tabular}{cccc}
\hline Product & $\mathrm{R}$ & $\mathrm{X}$ & Yield (\%) \\
\hline $\mathbf{5 a}$ & $\mathrm{Ph}$ & $\mathrm{F}$ & 74 \\
$\mathbf{5 b}$ & $\mathrm{Ph}$ & $\mathrm{Cl}$ & 70 \\
$\mathbf{5 c}$ & $\mathrm{Ph}$ & $\mathrm{Br}$ & 80 \\
$\mathbf{5 d}$ & $\mathrm{Ph}$ & $\mathrm{NO}_{2}$ & 63 \\
\hline
\end{tabular}

\section{Scheme 1}

The best molar ratio for the reaction between lithium acetylenides and enaminones was 1:1, respectively. Lower yields were found when other molar ratios were used. In order to improve the product yields, we used 2 mol-equiv of boron trifluoride diethyl etherate. Based on the results, ${ }^{8}$ the use of this Lewis acid effectively contributed to the improvement of yields of reactions with acetylenide derivatives. The use of catalytic amounts or one mol-equiv of Lewis acid did not afford satisfactory transformations, leading to enynes $\mathbf{4 , 5}$ with low yields. These facts are in accordance with the reaction mechanism proposed, where two boron trifluoride diethyl etherate molecules are presente in the transition state (Scheme 2). 


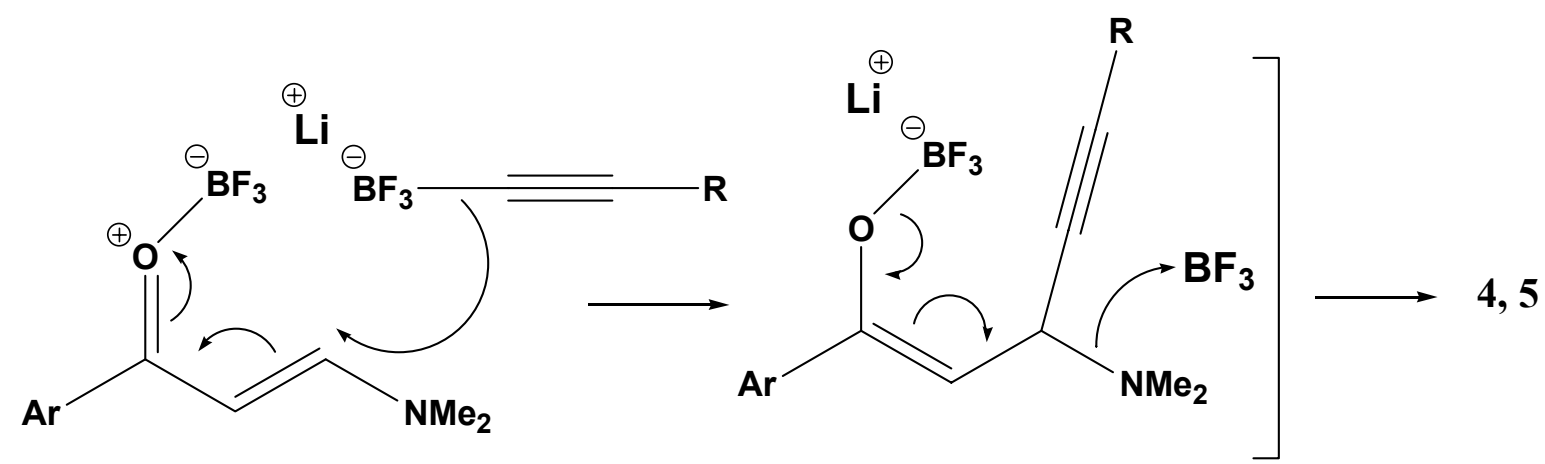

\section{Scheme 2}

Enynes 4,5 were identified by ${ }^{1} \mathrm{H}$ and ${ }^{13} \mathrm{C}$ NMR spectroscopy. In the ${ }^{1} \mathrm{H}$ NMR spectra, these products showed sets of signals corresponding to the proposed structures. Their analysis showed that the double bond of products $\mathbf{4 , 5}$ retained the $E$-configuration but with vicinal coupling constants around ${ }^{3} J_{\mathrm{H} 2-\mathrm{H} 3}=15 \mathrm{~Hz}$. The vinylic hydrogens which showed chemical shifts at the ranges of $\delta$ 5.65-5.68 ppm (H3) and $\delta 7.76-7.79 \mathrm{ppm}(\mathrm{H} 2)$ on the enaminones $\mathbf{3},{ }^{13}$ were found to show chemical shifts at the ranges of $\delta 6.89-7.17 \mathrm{ppm}$ and $\delta 7.13-7.46 \mathrm{ppm}$ for $\mathrm{H} 3$ and $\mathrm{H} 2$, respectively after the addition of acetylenides $\mathbf{1 , 2}$. Thus, one can conclude that the triple bond presented a deshielding effect for the hydrogen $\mathrm{H} 3$ at around 1.2-1.5 ppm and on the other hand, their electronic effect was to shield the $\mathrm{H} 2$ at around $0.3-0.6 \mathrm{ppm}$.

The ${ }^{13} \mathrm{C}$ NMR spectra of enynes $\mathbf{4 , 5}$ contained sets of signals corresponding to the postulated structure. The compounds 4 a-d presented chemical shifts of acetylenic carbons on average at $\delta$ 79.0 ppm and $\delta 102.7$ ppm for C4 and C5, respectively, while compounds 5a-d seemed to show these signals on average at $\delta 87.6 \mathrm{ppm}$ and $\delta 100.0 \mathrm{ppm}$. The signals assigned for vinylic carbons $\mathrm{C} 2$ and $\mathrm{C} 3$ presented chemical shifts on average at $\delta 126.6 \mathrm{ppm}$ and $\delta 132.5 \mathrm{ppm}$ for $\mathrm{C} 3$ and $\mathrm{C} 2$, respectively. The remaining signals presented typical ${ }^{13} \mathrm{C}$ chemical shifts of carbonyl, alkyl and aryl structures.

\section{Conclusions}

In summary, we have shown that in the presence of boron trifluoride diethyl etherate that regiospecific 1,4-addition of lithium acetylides can be carried out with a variety of $\beta$ dimethylaminovinyl ketones in moderate to good yield. The use of 2 mol-equiv of this Lewis acid was required to improve the product yields, as their presence in lower amounts reduced the reaction rate and the yields of enynes 4a-d, 5a-d. Future work will focus on utilizing this chemistry to prepare novel enynes-containing substances of pharmacological interest. 


\section{Experimental Section}

General Procedures. Unless otherwise indicated, all common reagents were used as obtained from commercial suppliers without further purification. The solvents were dried and purified according to recommended procedures. ${ }^{17}$ All melting points were determined on a MQAPF-301 (Microquímica Inc.) apparatus and are uncorrected. ${ }^{1} \mathrm{H}$ and ${ }^{13} \mathrm{C}$ NMR spectra were acquired on a Bruker DPX 400 spectrometer $\left({ }^{1} \mathrm{H}\right.$ at $400.13 \mathrm{MHz}$ and ${ }^{13} \mathrm{C}$ at $\left.100.62 \mathrm{MHz}\right)$ or on a Bruker DPX 200 spectrometer $\left({ }^{1} \mathrm{H}\right.$ at $200.13 \mathrm{MHz}$ and ${ }^{13} \mathrm{C}$ at $50.32 \mathrm{MHz}$ ), with $5 \mathrm{~mm}$ sample tubes, $300 \mathrm{~K}$, digital resolution $\pm 0.01 \mathrm{ppm}$, in $\mathrm{CDCl}_{3}$ using TMS as internal reference. Mass spectra were registered in a HP 5973 MSD connected to a HP 6890 GC and interfaced by a Pentium PC. IR spectra were obtained with a Bruker Tensor 27 spectrometer using films or $\mathrm{KBr}$ pellets of the compounds. The CHN elemental analyses were performed on a Perkin-Elmer $2400 \mathrm{CHN}$ elemental analyzer (Federal University of Rio Grande do Sul, UFRGS/ Brazil). Yields listed are of purified compounds.

\section{General procedure for synthesis of compounds 4a-d, 5a-d}

1.6 M Solution of BuLi in hexane $(5 \mathrm{mmol}, 3.12 \mathrm{~mL})$ and under argon was added dropwise to a stirred solution of acetylene $\mathbf{1 , 2},(5 \mathrm{mmol})$ in dry THF $(10 \mathrm{~mL})$ at $-20^{\circ} \mathrm{C}$. After stirring for 30 minutes, a solution of $\beta$-dimethylaminovinyl ketones 3a-d $(5 \mathrm{mmol})$ solubilized in THF $(3 \mathrm{~mL})$ and $\mathrm{BF}_{3} \bullet \mathrm{Et}_{2} \mathrm{O}(2.64 \mathrm{~mL}, 10 \mathrm{mmol})$ was added at $-15^{\circ} \mathrm{C}$, over approximately $2 \mathrm{~min}$. The mixture was stirred for 2 hours, and then after warming at room temperature was stirred for 16 hours. The solvent was removed under vacuum, and the crude product was solubilized in $\mathrm{CHCl}_{3}(50 \mathrm{~mL})$. The organic phase was washed with distillated water $(3 \times 15 \mathrm{~mL})$. For all products, the solution was dried with sodium sulfate anhydride, filtered and the solvent was removed by vacuum. The crude product was chromatographed (silica flash), with $5 \%$ ethyl acetate in hexane.

(E)-1-(4-Fluorophenyl)-dec-2-en-4-yn-1-one (4a). Yield $3.5 \mathrm{mmol}$ (70\%); oil; ${ }^{1} \mathrm{H}$ NMR (200 $\left.\mathrm{MHz}, \mathrm{CDCl}_{3}\right) \delta(J, \mathrm{~Hz}) 0.87$ (3H, t, $\left.J=7.9, \mathrm{H} 10\right), 1.29$ (6H, m, H7, H8, H9), 2.41 (2H, dt, $J=$ 7.0, 2.3, H6), 6.89 (1H, dt, $J=15.4,2.3, \mathrm{H} 3), 7.13(1 \mathrm{H}, \mathrm{d}, J=15.4, \mathrm{H} 2), 7.17$ - 7.90 (4 H, m, Ar); ${ }^{13} \mathrm{C}$ NMR (100 MHz, $\left.\mathrm{CDCl}_{3}\right) \delta\left(J_{\mathrm{C}-\mathrm{F}}, \mathrm{Hz}\right) 13.8$ (C10), 19.8 (C6), 22.1 (C9), 28.0 (C7), 31.0 (C8), 79.0 (C4), 102.0 (C5), 126.0 (C3), 132.0 (C2), 187.2 (C1), 133.7 (d, $\left.{ }^{4} J_{\mathrm{C}-\mathrm{F}}=2.8, \mathrm{Ar}\right), 130.5$ $\left(\mathrm{d},{ }^{3} J_{\mathrm{C}-\mathrm{F}}=9, \mathrm{Ar}\right), 115.7\left(\mathrm{~d},{ }^{2} J_{\mathrm{C}-\mathrm{F}}=21.8, \mathrm{Ar}\right), 165.0\left(\mathrm{~d},{ }^{1} J_{\mathrm{C}-\mathrm{F}}=255.0, \mathrm{Ar}\right) ; \mathrm{MS} m / z(\%) 244\left(\mathrm{M}^{+}\right.$, 40), 201 (66), 123 (100), 95 (98); IR (film, v, cm ${ }^{-1}$ ) 2932, 2212, 1662, 1599. Anal. Calcd. for $\mathrm{C}_{16} \mathrm{H}_{17} \mathrm{FO}$ : C, 78.66\%; H, 7.01. Found: C, 78.87\%; H, $7.25 \%$.

(E)-1-(4-Chlorophenyl)-dec-2-en-4-yn-1-one (4b). Yield 3.75 mmol (75\%); oil; ${ }^{1} \mathrm{H}$ NMR (200 $\left.\mathrm{MHz}, \mathrm{CDCl}_{3}\right) \delta(J, \mathrm{~Hz}) 0.92(3 \mathrm{H}, \mathrm{t}, J=6.9, \mathrm{H} 10), 1.28(6 \mathrm{H}, \mathrm{m}, \mathrm{H} 7, \mathrm{H} 8, \mathrm{H} 9), 2.41$ ( $2 \mathrm{H}, \mathrm{dt}, J=$ 6.9, 2.1, H6), 6.90 (1H, dt, $J=15.4,2, \mathrm{H} 3), 7.22(1 \mathrm{H}, \mathrm{d}, J=15.4, \mathrm{H} 2), 7.44$ (2H, d, $J=8.7, \mathrm{Ar})$, 7.89 (2 H, d, $J=8.7, \mathrm{Ar}) ;{ }^{13} \mathrm{C}$ NMR (100 MHz, $\left.\mathrm{CDCl}_{3}\right) \delta 13.8$ (C10), 19.8 (C6), 22.1 (C9), 27.9 (C7), 31.1 (C8), 79.0 (C4), 102.2 (C5), 126.7 (C3), 131.9 (C2) 128.8, 129.8, 135.7, 139.4, (6C, Ar) 187.9 (C1); MS m/z (\%) $260\left(\mathrm{M}^{+}, 14\right), 225$ (80), 139 (100), 111 (61); IR (film, v, cm ${ }^{-1}$ ) 
2931, 2212, 1661, 1594. Anal. Calcd. for $\mathrm{C}_{16} \mathrm{H}_{17} \mathrm{ClO}: \mathrm{C}, 73.70 \%$; H, 6.57\%. Found: $\mathrm{C}, 73.93 \%$; $\mathrm{H}, 6.76 \%$.

(E)-1-(4-Bromophenyl)-dec-2-en-4-yn-1-one (4c). Yield $3.91 \mathrm{mmol}$ (78\%); oil; ${ }^{1} \mathrm{H}$ NMR (200 $\left.\mathrm{MHz}, \mathrm{CDCl}_{3}\right) \delta(J, \mathrm{~Hz}) 0.91(3 \mathrm{H}, \mathrm{t}, J=6.7, \mathrm{H} 10), 1.35(6 \mathrm{H}, \mathrm{m}, \mathrm{H} 7, \mathrm{H} 8, \mathrm{H} 9), 2.40$ ( 2H, dt, $J=$ 6.9, 2.1, H6), 6.89 (1H, dt, $J=15.4,2.3, \mathrm{H} 3), 7.21(1 \mathrm{H}, \mathrm{d}, J=15.4, \mathrm{H} 2), 7.55(2 \mathrm{H}, \mathrm{d}, J=8.5$, $\mathrm{Ar}), 7.79(2 \mathrm{H}, \mathrm{d}, J=8.5, \mathrm{Ar}) ;{ }^{13} \mathrm{C} \mathrm{NMR}\left(100 \mathrm{MHz}, \mathrm{CDCl}_{3}\right) \delta 13.8$ (C10), 19.8 (C6), 22.0 (C9), 27.9 (C7), 30.9 (C8), 79.1 (C4), 102.6 (C5), 128.0 (C3), 135.9 (C2) 128.1, 129.8, 131.7, 137.2 (6C, Ar), 187.7 (C1); MS m/z (\%) $304\left(\mathrm{M}^{+}, 11\right), 306\left(\mathrm{M}^{+}+2,11\right), 225$ (100), 183 (71), 155 (70); IR (film, $v, \mathrm{~cm}^{-1}$ ) 2930, 2212, 1660, 1592. Anal. Calcd. for $\mathrm{C}_{16} \mathrm{H}_{17} \mathrm{BrO}$ : C, 62.96\%; H, 5.61\%. Found: C, 63.20\%; H, 5.90\%.

(E)-1-(4-Nitrophenyl)-dec-2-en-4-yn-1-one (4d). Yield $3.25 \mathrm{mmol}$ (65\%); oil; ${ }^{1} \mathrm{H}$ NMR (200 $\left.\mathrm{MHz}, \mathrm{CDCl}_{3}\right) \delta(J, \mathrm{~Hz}) 0.94(3 \mathrm{H}, \mathrm{t}, J=6.9, \mathrm{H} 10), 1.44(6 \mathrm{H}, \mathrm{m}, \mathrm{H} 7, \mathrm{H} 8, \mathrm{H} 9), 2.44$ (2H, dt, $J=7$, $J=2, \mathrm{H} 6), 6.91(1 \mathrm{H}, \mathrm{dt}, J=15.4,2.3, \mathrm{H} 3), 7.20(1 \mathrm{H}, \mathrm{d}, J=15.4, \mathrm{H} 2), 8.81(2 \mathrm{H}, \mathrm{d}, J=8.5, \mathrm{Ar})$, $8.32(2 \mathrm{H}, \mathrm{d}, J=8.7, \mathrm{Ar}) ;{ }^{13} \mathrm{C}$ NMR (100 MHz, $\left.\mathrm{CDCl}_{3}\right) \delta 13.8$ (C10), 19.9 (C6), 22.1 (C9), 27.8 (C7), 31.0 (C8), 79.1 (C4), 104.2 (C5), 128.3 (C3), 131.2 (C2), 123.7, 129.2, 142.4, 150.5 (6C, Ar), 187.7 (C1); MS m/z (\%) $271\left(\mathrm{M}^{+}, 14\right), 228$ (100), 215 (40), 150 (66), 121 (28); IR (film, $v$, $\mathrm{cm}^{-1}$ ) 2930, 2207, 1698, 1601. Anal. Calcd. for $\mathrm{C}_{16} \mathrm{H}_{17} \mathrm{NO}_{3}$ : C, 70.83\%; H, 6.32\%; N, 5.16\%. Found: C, 71.16\%; H, 6.91\%; N, 5.37\%.

(E)-1-(4-Fluorophenyl)-5-phenylpent-2-en-4-yn-1-one (5a). Yield $3.72 \mathrm{mmol}$ (74\%); mp 63$64{ }^{\circ} \mathrm{C} ;{ }^{1} \mathrm{H}$ NMR $\left(200 \mathrm{MHz}, \mathrm{CDCl}_{3}\right) \delta(J, \mathrm{~Hz}) 7.03(1 \mathrm{H}, \mathrm{d}, J=15.4, \mathrm{H} 3), 7.46(1 \mathrm{H}, \mathrm{d}, J=15.4$, $\mathrm{H} 2)$, 7.93-6.97 (9H, m, Ph, Ar); ${ }^{13} \mathrm{C}$ NMR (50 MHz, CDCl $) \delta\left(J_{\mathrm{C}-\mathrm{F}}, \mathrm{Hz}\right) 87.6$ (C4), 99.5 (C5), $115.7\left(\mathrm{~d},{ }^{2} J_{\mathrm{C}-\mathrm{F}}=22.2, \mathrm{Ar}\right), 125.2(\mathrm{C} 3), 131.3\left(\mathrm{~d},{ }^{3} J_{\mathrm{C}-\mathrm{F}}=9.4, \mathrm{Ar}\right), 122.1,128.4,129.3,131.9(6 \mathrm{C}$, $\mathrm{Ph}), 132.4(\mathrm{C} 2), 133.4$ (d, $\left.{ }^{4} J_{\mathrm{C}-\mathrm{F}}=3,03, \mathrm{Ar}\right), 165.6\left(\mathrm{~d},{ }^{1} J_{\mathrm{C}-\mathrm{F}}=255.3, \mathrm{Ar}\right), 186.9(\mathrm{C} 1) ; \mathrm{MS} m / z(\%)$ $250\left(\mathrm{M}^{+}, 98\right) ; 155$ (28); 220 (100); 127 (80); IR (KBr, v, $\left.\mathrm{cm}^{-1}\right)$ 2212, 1662, 1599. Anal. Calcd. for $\mathrm{C}_{17} \mathrm{H}_{11} \mathrm{FO}$ : C, $81.59 \%$; $\mathrm{H}, 4.43 \%$. Found: $\mathrm{C}, 81.72 \%$; H, 4.64\%.

(E)-1-(4-Chlorophenyl)-5-phenylpent-2-en-4-yn-1-one (5b). Yield $3.52 \mathrm{mmol}$ (70\%); mp 85$87{ }^{\circ} \mathrm{C} ;{ }^{1} \mathrm{H}$ NMR $\left(200 \mathrm{MHz}, \mathrm{CDCl}_{3}\right) \delta(J, \mathrm{~Hz}) 7,13(1 \mathrm{H}, \mathrm{d}, J=15.4, \mathrm{H} 3), 7.39(1 \mathrm{H}, \mathrm{d}, J=15.4$, $\mathrm{H} 2), 795-7.35$ (9H, m, Ph, Ar); ${ }^{13} \mathrm{C}$ NMR (100 MHz, $\left.\mathrm{CDCl}_{3}\right) \delta 87.6$ (C4), 99.7 (C5), 125.5 (C3), 129.4, 122.1, 128.4, 128.9, 129.8, 132.0, 135.1, 139.0 (12C, Ph, Ar), 132.4 (C2), 187.4 (C1); MS $\mathrm{m} / z(\%) 266\left(\mathrm{M}^{+}, 19\right), 231$ (100), 111 (28), 202 (52), 77 (23); IR (KBr, $v, \mathrm{~cm}^{-1}$ ) 2199, 1652, 1585. Anal. Calcd. for $\mathrm{C}_{17} \mathrm{H}_{11} \mathrm{ClO}$ : C, 76.55\%; H, 4.16\%. Found: $\mathrm{C}, 76.78 \%$; $\mathrm{H}, 4.40 \%$.

(E)-1-(4-Bromophenyl)-5-phenylpent-2-en-4-yn-1-one (5c). Yield 4.0 mmol (80\%); mp 91-93 ${ }^{\circ} \mathrm{C} ;{ }^{1} \mathrm{H}$ NMR $\left(200 \mathrm{MHz}, \mathrm{CDCl}_{3}\right) \delta(J, \mathrm{~Hz}) 7.12(1 \mathrm{H}, \mathrm{d}, J=15.4, \mathrm{H} 3), 7.36(1 \mathrm{H}, \mathrm{d}, J=15.4, \mathrm{H} 2)$, 7.8-7.34 (9H, m, Ph, Ar); ${ }^{13} \mathrm{C}$ NMR (50 MHz, $\left.\mathrm{CDCl}_{3}\right) \delta 87.6$ (C4), 99.8 (C5), 125.5 (C3), 122.3, 128.3, 128.4, 129.4, 129.9, 131.9, 132.0, 135.8 (12C, Ph, Ar), 132.2 (C2), 187.5 (C1); MS m/z (\%) $310\left(\mathrm{M}^{+}, 8\right), 312\left(\mathrm{M}^{+}+2,8\right), 231$ (100), 202 (71), 155 (28), 77 (52); IR (KBr, v, cm $\left.{ }^{-1}\right)$ 2197, 1651, 1586. Anal. Calcd. for $\mathrm{C}_{17} \mathrm{H}_{11} \mathrm{BrO}: \mathrm{C}, 65.62 \% ; \mathrm{H}, 3.56 \%$. Found: $\mathrm{C}, 65.79 \% ; \mathrm{H}, 3.74 \%$.

(E)-1-(4-Nitrophenyl)-5-phenylpent-2-en-4-yn-1-one (5d). Yield 3.15 mmol (63\%); mp 133$136^{\circ} \mathrm{C} ;{ }^{1} \mathrm{H}$ NMR $\left(200 \mathrm{MHz}, \mathrm{CDCl}_{3}\right) \delta(J, \mathrm{~Hz}) 7.17(1 \mathrm{H}, \mathrm{d}, J=15.4, \mathrm{H} 3), 7.40(1 \mathrm{H}, \mathrm{d}, J=15.4$, $\mathrm{H} 2), 8.37-7.35$ (9H, m, Ph); ${ }^{13} \mathrm{C}$ NMR (100 MHz, $\left.\mathrm{CDCl}_{3}\right) \delta 87.5$ (C4), 101.2 (C5), 127.1 (C3), 
123.8, 125.4, 128.5, 129.4, 129.7, 132.1, 141.9, 150.2 (12C, Ph, Ar), 131.9 (C2), 187.7 (C1); MS m/z (\%) $277\left(\mathrm{M}^{+}, 36\right), 231$ (54), 202 (100), 155 (18), 127 (90), 77 (95); IR (KBr, v, cm ${ }^{-1}$ ) 2188, 1657, 1604. Anal. Calcd. for $\mathrm{C}_{17} \mathrm{H}_{11} \mathrm{NO}_{3}$ : C, 73.64\%; H, 4.00\%; N, 5.05. Found: C, 73.80\%; H, $4.23 \% ; \mathrm{N}, 5.29 \%$.

\section{Acknowledgements}

The authors thank the Conselho Nacional de Desenvolvimento Científico e Tecnológico (CNPq/PADCT) and Fundação de Amparo à Pesquisa do Estado do Rio Grande do Sul (FAPERGS) for financial support. The fellowships from CNPq (M. Rossatto and F. A. Rosa), and CAPES (P. Machado) are also acknowledged.

\section{References}

1. (a) Feuerstein, M.; Chahen, L.; Doucet, H.; Santelli, M. Tetrahedron, 2006, 62, 112. (b) Silveira, C. C.; Braga, A. L.; Vieira, A. S.; Zeni, G. J. Org. Chem. 2003, 68, 662. (c) Nicolaou, K. C.; Smith, A. L. In Modern Acetylene Chemistry; Stang, P. J., Diederich, F., Eds.; VCH: Weinheim, 1995.

2. Konishi, M.; Ohkuma, H.; Matsumoto, K.; Tsuno, T.; Kamei, H.; Miyake, T.; Oki, T.; Kawaguchi, H.; Van Duyne, G. D.; Clardy, J. J. Antibiotics. 1989, 42, 1449.

3. Nussbaumer, P.; Leitner, L.; Marz, K.; Stutz, A. J. Med. Chem. 1995, 38, 1831.

4. De Luca, A.; Cozzi-Lepri, A.; Antinori, A.; Zaccarelli, M.; Bongiovanni, M.; Di Giambenedetto, S.; Marconi, P.; Cicconi, P.; Resta, F.; Grisorio, B.; Ciardi, M.; Cauda, R.; d'Arminio, A. M. Antiviral Ther. 2006, 11, 609.

5. Patrick, G. L. In An Introduction to Medicinal Chemistry; Eds.; Oxford: New York, 2001; p 203.

6. (a) Sonogashira, K.; Tohda, Y.; Hagihara, N. Tetrahedron Lett. 1975, 16, 4467. (b) Alami, M.; Linstrumelle, G. Tetrahedron Lett. 1991, 32, 6109. (c) Dieck, H. A.; Heck, F. R. J. Organomet. Chem. 1975, 93, 259. (d) Scott, W. J.; Peña, M. R.; Sward, K.; Stoessel, S. J.; Stille, J. K. J. Org. Chem. 1985, 50, 2302. (e) Alami, M.; Crousse, B.; Ferri, F. J. Organomet. Chem. 2001, 624, 114. (f) Takeuchi, R.; Tanabe, K.; Tanaka, S. J. Org. Chem. 2000, 65, 1558. (g) Organ, M. G.; Ghasemi, H.; Valente, C. Tetrahedron 2004, 60, 17489. (h) Gallagher, W. P.; Maleczka, R. E., Jr. J. Org. Chem. 2003, 68, 6775.

7. Andrew, R. J.; Mellor, J. M. Tetrahedron 2000, 56, 7261.

8. Martins, M. A. P.; Emmerich, D. J.; Pereira, C. M. P, Cunico, W.; Rossatto, M.; Zanatta, N.; Bonacorso, H. G. Tetrahedron Lett. 2004, 45, 4935; Martins, M. A. P.; Rossatto, M.; Frizzo, C. P.; Machado, P.; Pizzuti, L.; Emmerich, D.; Pereira, J. I.; Brondani, S.; Zanatta, N.; Bonacorso, H. G.; Flores, A. F. C. Lett. Org. Chem., 2007, 4, 146. 
9. (a) Colla, A.; Martins, M. A. P.; Clar, G.; Krimmer, S.; Fisher, P. Synthesis 1991, 6, 483; (b) Martins, M. A. P.; Zoch, A. N.; Flores, A. F. C.; Clar, G.; Zanatta, N.; Bonacorso, H. G. J. Heterocycl. Chem. 1995, 32, 739; (c) Martins M. A. P.; Bastos, G. P. Bonacorso, H. G.; Zanatta, N.; Flores, A .F. C.; Siqueira, G. M. Tetrahedron Lett. 1999, 40, 4309; (d) Bonacorso, H.G; Martins, M.A.P.; Bittencourt, S. R. T.; Lourega, R.V.; Zanatta, N.; Flores, A. F. C. J. Fluorine Chem. 1999, 99, 177.

10. Martins, M. A. P.; Cunico, W.; Pereira, C. M. P; Flores, A. F. C.; Bonacorso H. G.; Zanatta, N. Curr. Org. Synthesis 2004, 1, 391, and references therein.

11. (a) Martins, M. A. P.; Zanatta, N.; Bonacorso, H. G.; Rosa, F. A.; Claramunt, R. M.; García, M. A.; Santa María, M. D.; Elguero, J. Arkivoc 2006, iv, 29; (b) Martins, M. A. P.; Pereira, C. M. P.; Moura, S.; Fiss, G. F.; Frizzo, C. P.; Emmerich, D. J.; Zanatta, N.; Bonacorso, H. G. Arkivoc 2006, (xiii), 187.

12. (a) Chimichi, S.; Boccalini, M.; Hassan, M. M. M.; Viola, G.; Dall'Acqua, F.; Curini, M. Tetrahedron 2006, 62, 90. (b) Tseng, S-S.; Epstein, J. W.; Brabander, H. J.; Francisco, G. J. Heterocyclic Chem. 1987, 24, 837. (c) Reidlinger, C.; Dworczak, R.; Junek, H. Monatsh Chem. 1998, 129, 1207. (d) Al-Omram, F.; Elassar, A-Z. A.; El-Khair, A. A. Tetrahedron 2001, 57, 10163.

13. Rosa, F. A. Master Dissertation, Federal University of Santa Maria, Brazil, 2005.

14. (a) Gorbunova, M. G.; Gerus, I. I.; Kukher, V. P. J. Fluorine Chem., 1993, 65, 25; (b) Bravo, P.; Bruche, L.; Farina, A.; Gerus, I. I.; Kolycheva, M. T.; Kukhar, V. P.; Meille, S.V.; Viani, F. J. Chem. Soc., Perkin Trans. 1 1995, 1667.

15. Kruchok, I.S.; Gerus, I.I.; Kukhar, V.P. Tetrahedron 2000, 56, 6533.

16. (a) Kabalka, G.W.; Mereddy, A.R. Organometallics 2004, 23, 4519; (b) Molander, G.A. and Ham, J. Org. Lett. 2006, 8, 2031.

17. Perrin, D. D.; Armarego, W. L. F. Purification of Laboratory Chemicals, Eds.; Pergamon: Oxford, 1998. 